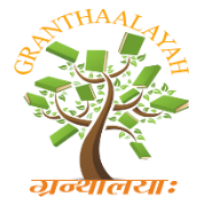

\author{
INTERNATIONAL JOURNAL OF RESEARCH - \\ GRANTHAALAYAH \\ A knowledge Repository
}

Science

\title{
ENERGY DETECTION IN THE FORM OF LIGHT RADIATION AT END OF HUMAN BLOOD COAGULATION CASCADE- THE OPTICAL ABSORPTION OF WATER VS. FIBRIN BURST ENERGY RELEASE
}

\author{
Abraham A. Embi Bs MBA *1 \\ *1 13442 SW 102 Lane, Miami, Florida USA, 33186
}

\begin{abstract}
The human blood coagulation cascade had been extensively researched from a biochemistry and molecular perspective. The purpose of this manuscript is to introduce a biophysical phenomenon detected via optical microscopy at the end of the human blood coagulation cascade. This could be described as a sudden energy event in the form of light radiation observed once blood tissue movement stops being attracted to metal iron filings or carbon based graphite particles used as sentinels. Upon close examination of video recordings, the sudden movements of iron particles images coincided with light at the end of the coagulation cascade. A literature search confirmed that both metal filings and graphite particles to possess excellent electrical conductivity. A biophysical light radiation event discharge is hypothesized as result of a burst in the conversion of fibrinogen to fibrin signaling the end of a coagulation cycle; perhaps combined with a piezoelectric effect induced by a sudden clumping of RBCs, or from the optical absorption or water.

Method: Metal iron filings or graphite particles were randomly sprinkled on fresh TIBS preparations. The sample was then readily focused and selected particles chosen for video analysis. Equipment used was a video microscope Celestron Model \# 44348, glass slides and author's blood drops. The data was digitally transferred and stored in an Apple computer photo application for further review.

Results: When metal iron filings or graphite particles were sprinkled on freshly prepared TIBS slides, video analysis show light radiation emitted at the end of the blood coagulation cascade.

Discussion: Since the light radiation emission occurred at the end of the blood coagulation cycle, it is theorized that blood tissue compression could induce piezoelectricity coinciding with energy released by a fibrin burst, or by the optical absorption of water.

Keywords: In Vivo Blood Smears; TBIS; Blood Coagulation Cascade; Piezoelectric Effect; Metal Iron Conductivity; Graphite Conductivity; Fibrin Burst Light Radiation; Optical Absorption of Water.

Cite This Article: Abraham A. Embi Bs MBA. (2019). "ENERGY DETECTION IN THE FORM OF LIGHT RADIATION AT END OF HUMAN BLOOD COAGULATION CASCADE- THE OPTICAL ABSORPTION OF WATER VS. FIBRIN BURST ENERGY RELEASE." International Journal of Research - Granthaalayah, 7(9), 200-212. https://doi.org/10.5281/zenodo.3472760.
\end{abstract}




\section{Introduction}

An optical microscopy method to study in vivo whole fresh human blood drops physical responses to bio-magnetically active and inert material were recently introduced and described $(1,2,3)$. The method demonstrated that when whole blood is smeared onto a glass slide, there is a time window or Wet Time (WT) of approximately $60 \pm 20$ seconds for a complete water evaporation of the smeared blood tissue. During the WT the human blood components seem to retain their in vivo physical properties, ie: blood cells are documented flowing and maintaining their intrinsic laminar flows. During the WT, foreign bio-magnetically inert material ie: salt (Fig. 1) or paramagnetic active ie: pepper (Fig. 1A) placed on the slide will either scatter blood components or concentrically repulse the blood tissue.

\section{Temporary In Vivo Blood Smears}

As soon as a blood smear is done, water evaporation is observed to start concentrically at the edge of the slide and ending approximately at the center. Molecularly, as the WT comes to an end, the ultimate effect of the blood coagulation cascade will eventually reach a point where the action of the protease enzyme (thrombin) on fibrinogen causes a fibrin burst. The polymerized fibrin together with platelets forms a hemostatic plug or clot $(4,5,6,7,8)$.

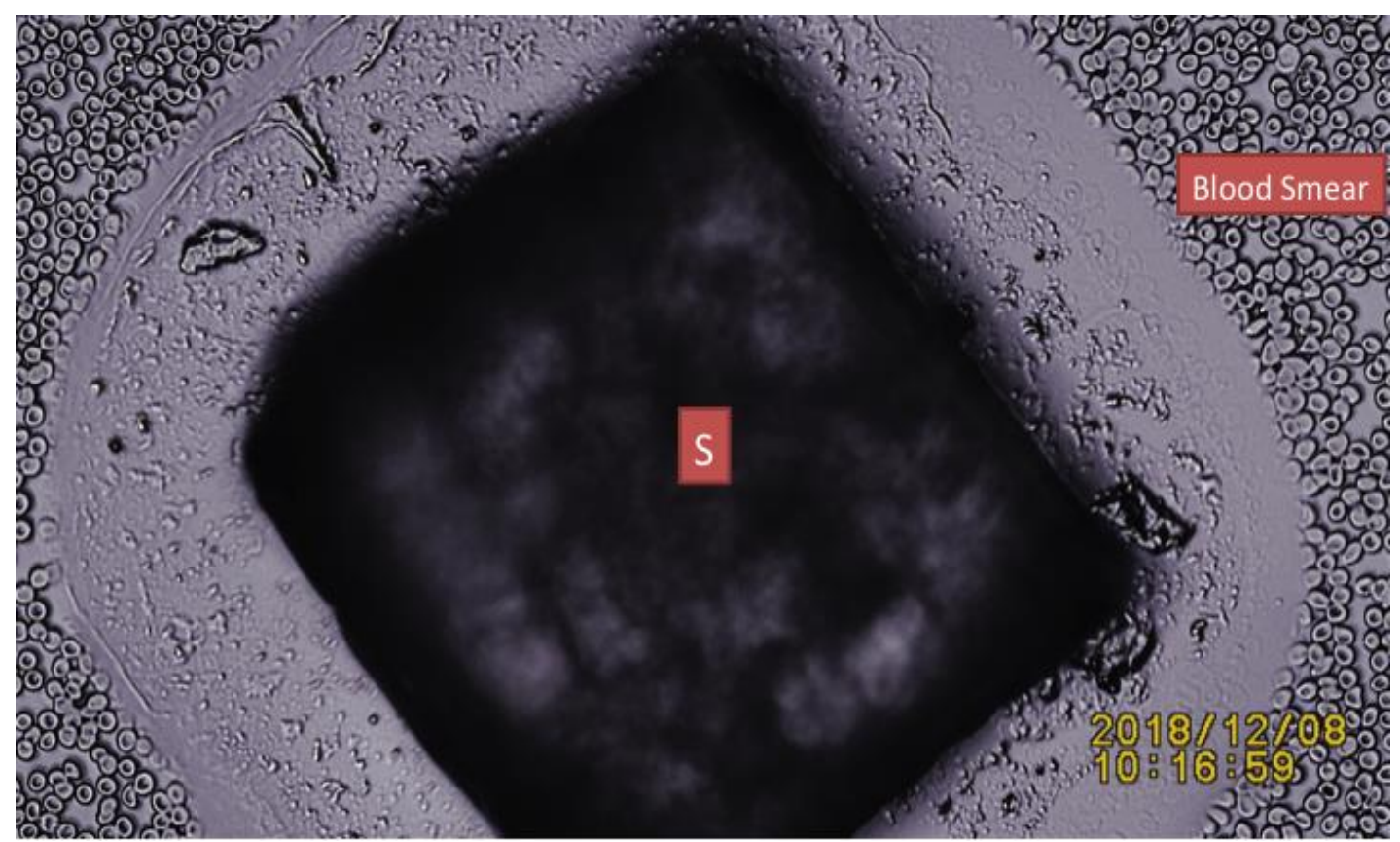

Figure 1: $n=1$ Amplified image of magnetically inert salt crystal in wet blood smear. Notice the symmetry seen on the blood being displaced by the salt. 


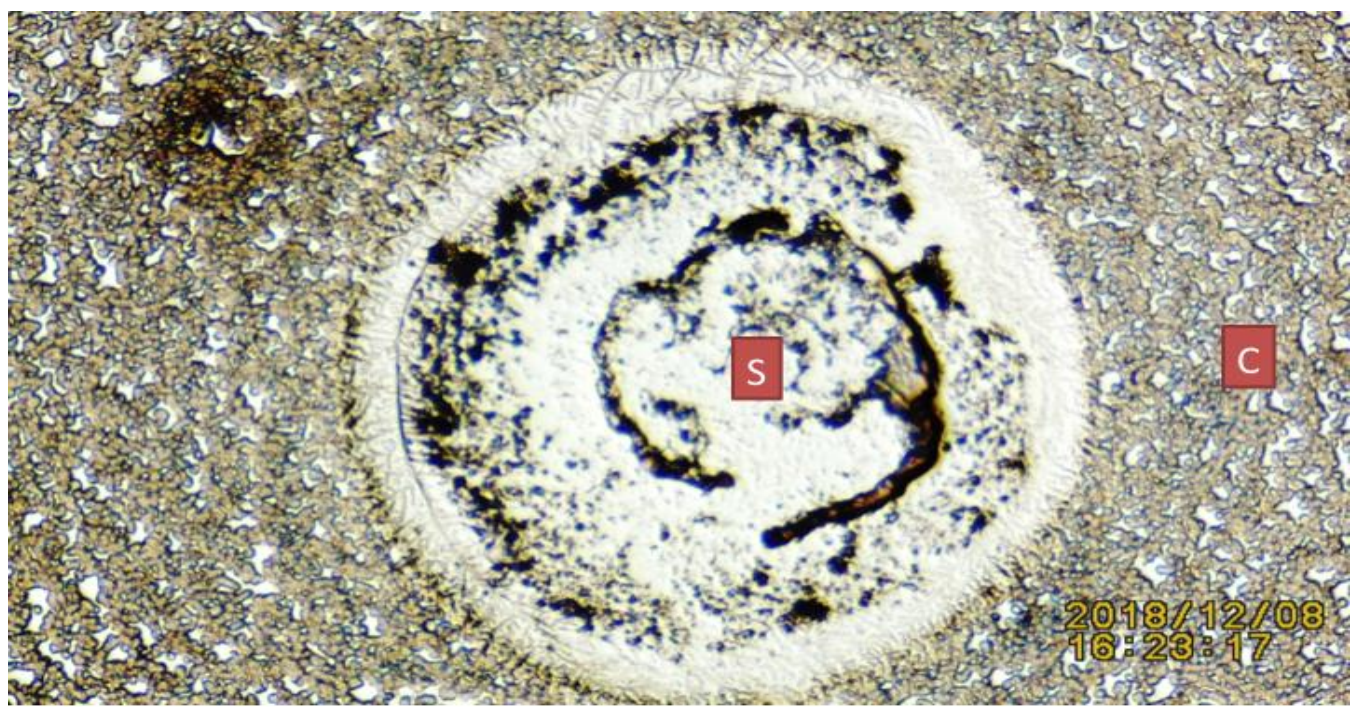

Figure 1A: The actual grain of salt now removed. Showing asymmetrical blood repulsion from a magnetically inert grain of salt. $\mathrm{S}=$ Debris after removal of salt grain. $\mathrm{C}=$ Coagulated Blood.

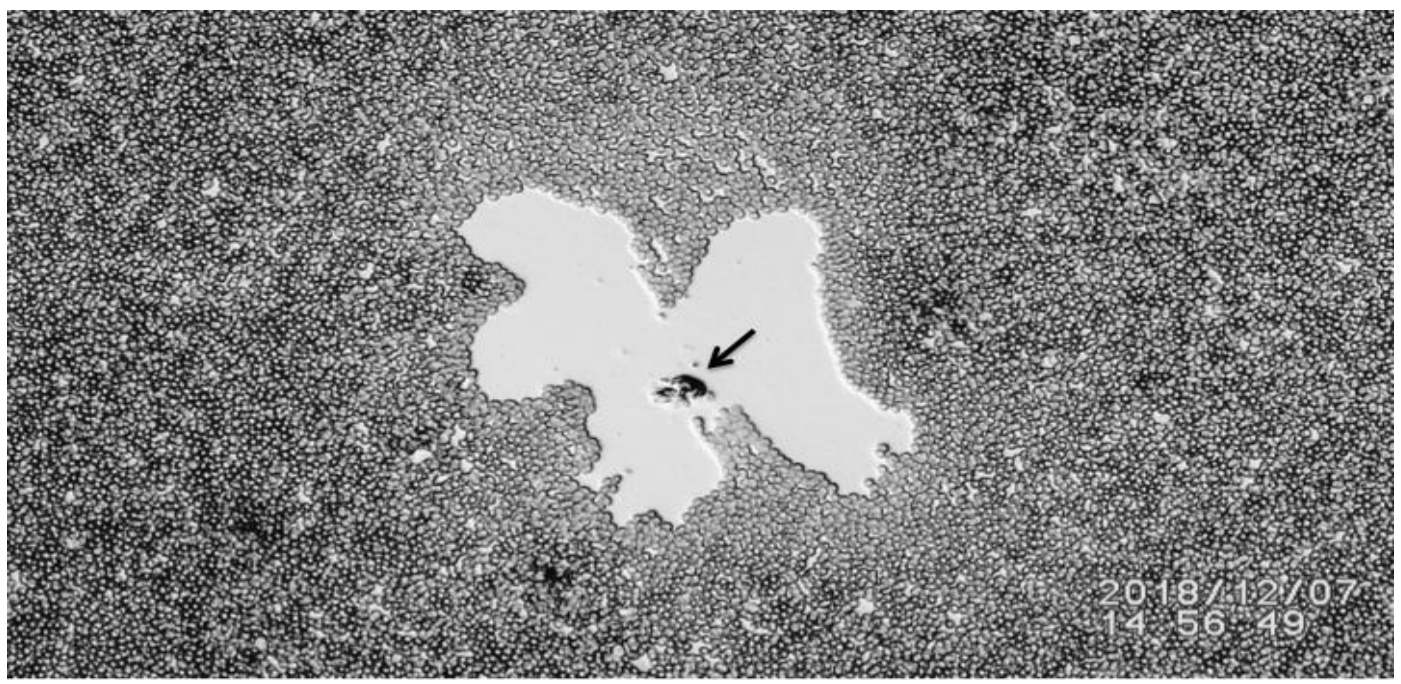

Figure 2: Showing Image of small magnetically active pepper flake asymmetrically displacing a wet blood smear.

Reproduced from: Embi AA (2018) Biomagnetism as Factor in Red Blood Cells Deformations. IJRG, 6 (12) 46-57. https://doi.org/10.5281/zenodo.2528404,

\section{Materials and Methods}

\section{Description of Experiments Performed The Temporary In Vivo Blood Smear or (TIBS)}

\section{Methodology}

A finger stick allowed for the milking of two drops of blood, then placed on a clean $25 \times 75 \times 1 \mathrm{~mm}$ glass slide. The mechanical smear was done as per published instructions from the USA center for disease control (9). There is a time window (TW) of approximately $60 \pm 20$ seconds for a complete 
water evaporation of the smeared blood tissue. The placement of iron metal filings or carbon base graphite particles (approximately midway the TW) were photographed and video recorded by using a Celestron Video Microscope Model \#44348 and a MacBook Pro Apple Computer Photo Application for digital images storage for further evaluation.

\section{Time Constraints}

Since time is of the essence (Less that 80 seconds) to successfully place the foreign particles on the evaporating smear, the following steps are suggested:

- Preset the video microscope into the video mode.

- Have the exogenous particles easily accessible for placement.

- Puncture a finger digit and milk blood.

- Place two blood drops on a slide and prepare a fresh smear.

- Sprinkle the exogenous particles in the center of the evaporating smear.

- Readily mount the smear on the microscope-viewing platform and locate suitable particle(s).

- Change the lens magnification for optimal viewing details.

- Begin video recording, with the goal of documenting the evaporation process.

- Select images for analysis and possible presentation.

- Repeat above if unsuccessful.

The placing of Metal Iron Filings and Graphite on TIBS

A total of $15+$ experiments were done where metal iron filings were randomly placed on TIBS $_{\mathrm{s}}$. And a total of 2 with graphite particles.

\section{Results}

\section{Iron Metal Filings in TIBS \\ Data Presented}

Results from experiments are qualitative. In other words, there is zero quantification to report, images are shown as follows:

\section{Metal Iron Filing in TIBS}

The figures below represent typical pre and post experiments of the interaction of in vivo human whole blood particles (Red Blood Cells, White Blood Cells and Platelets) with metal iron filings (Figs 3,4,5,6). 


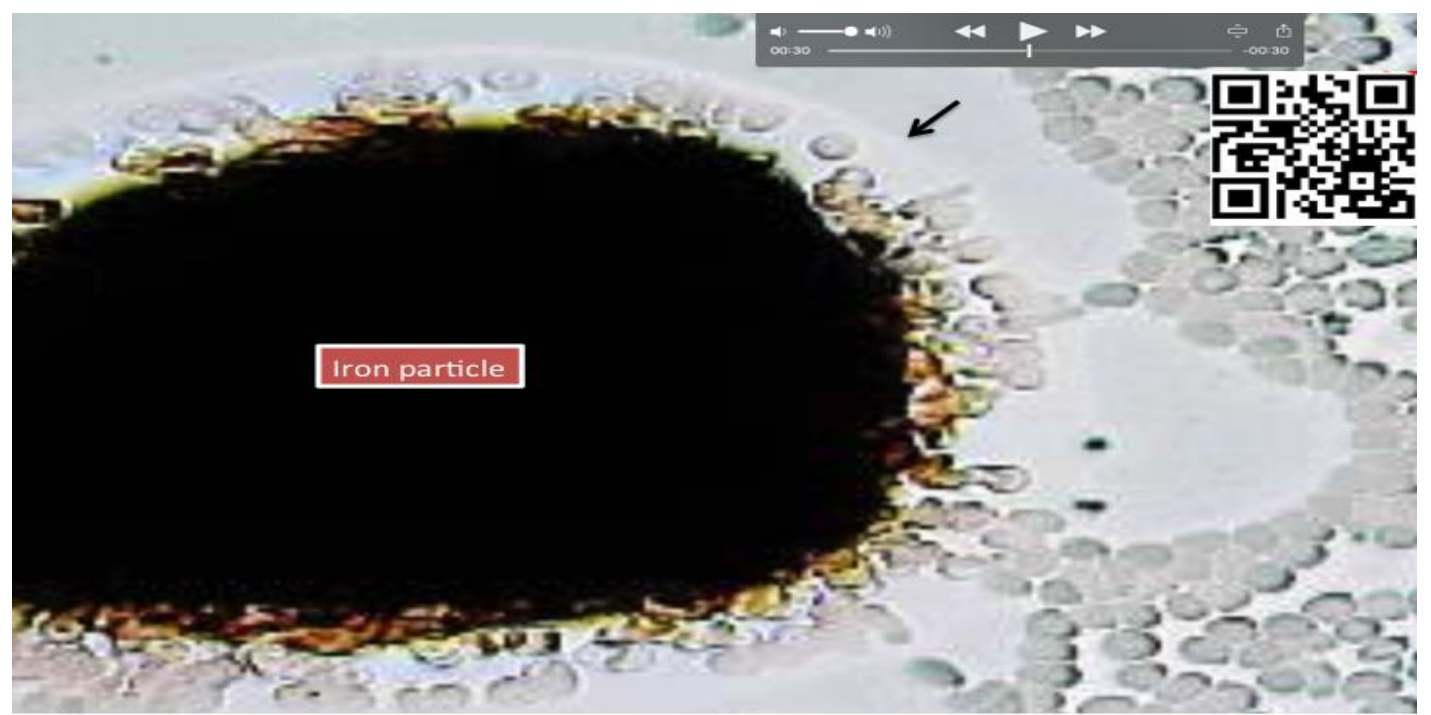

Figure 3: Frame 00:30" of video recording. Iron particle in TIBS showing light flashes while blood coagulation is underway. Black Arrow pointing at Halo denoting electrical discharges.

Please link to: https://youtu.be/0nItbtbpxVg

Or Scan QR Code on top right of image.

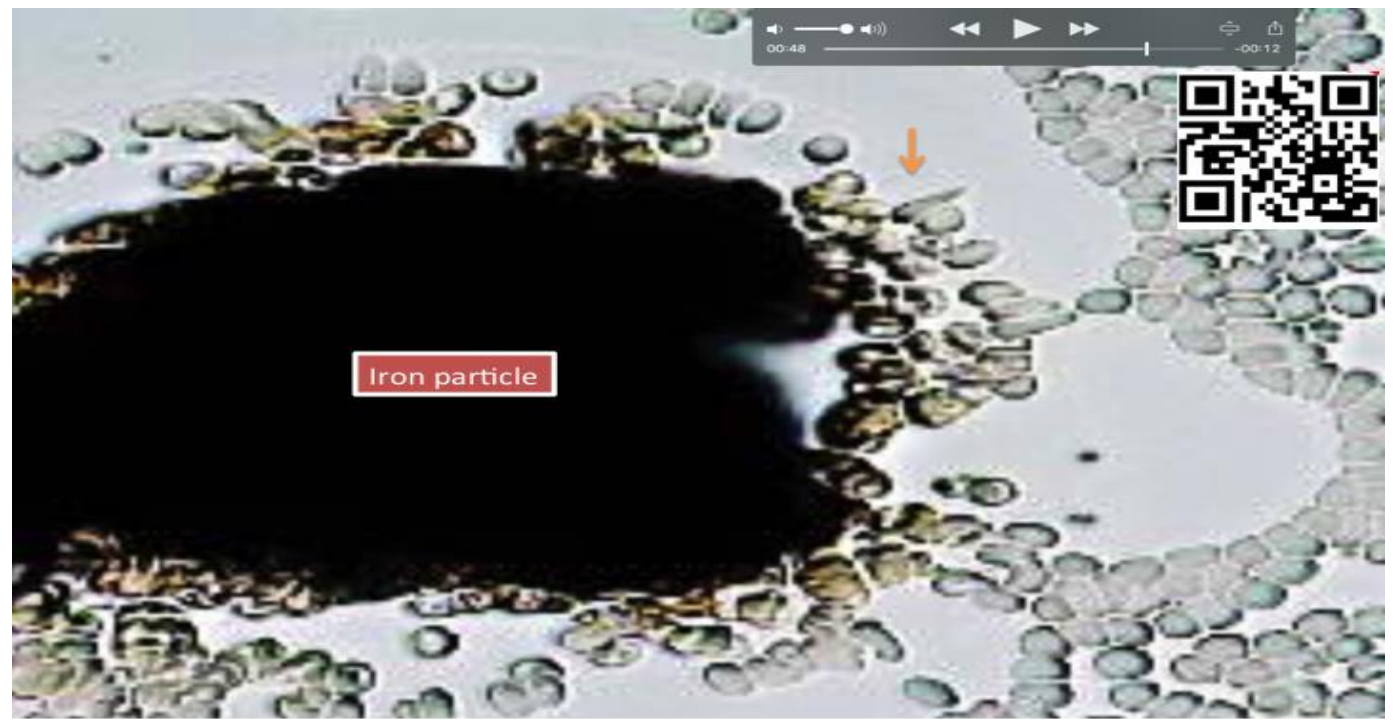

Figure 4: Frame 00:48" of video recording. Iron particle in TIBS after cessation of light flashes, possibly indicating end of coagulation theorized to follow contraction. Orange arrow pointing at compressed Red Blood Cell. Please link to: https://youtu.be/0nItbtbpxVg

Or Scan QR Code on top right of image.

Additional example of light radiation emission as the RBCs are attracted towards the center of metal iron filings. To appreciate the light radiation emission suggested is to move the cursor at a faster speed between 00.05" and 00:10" of recording. 


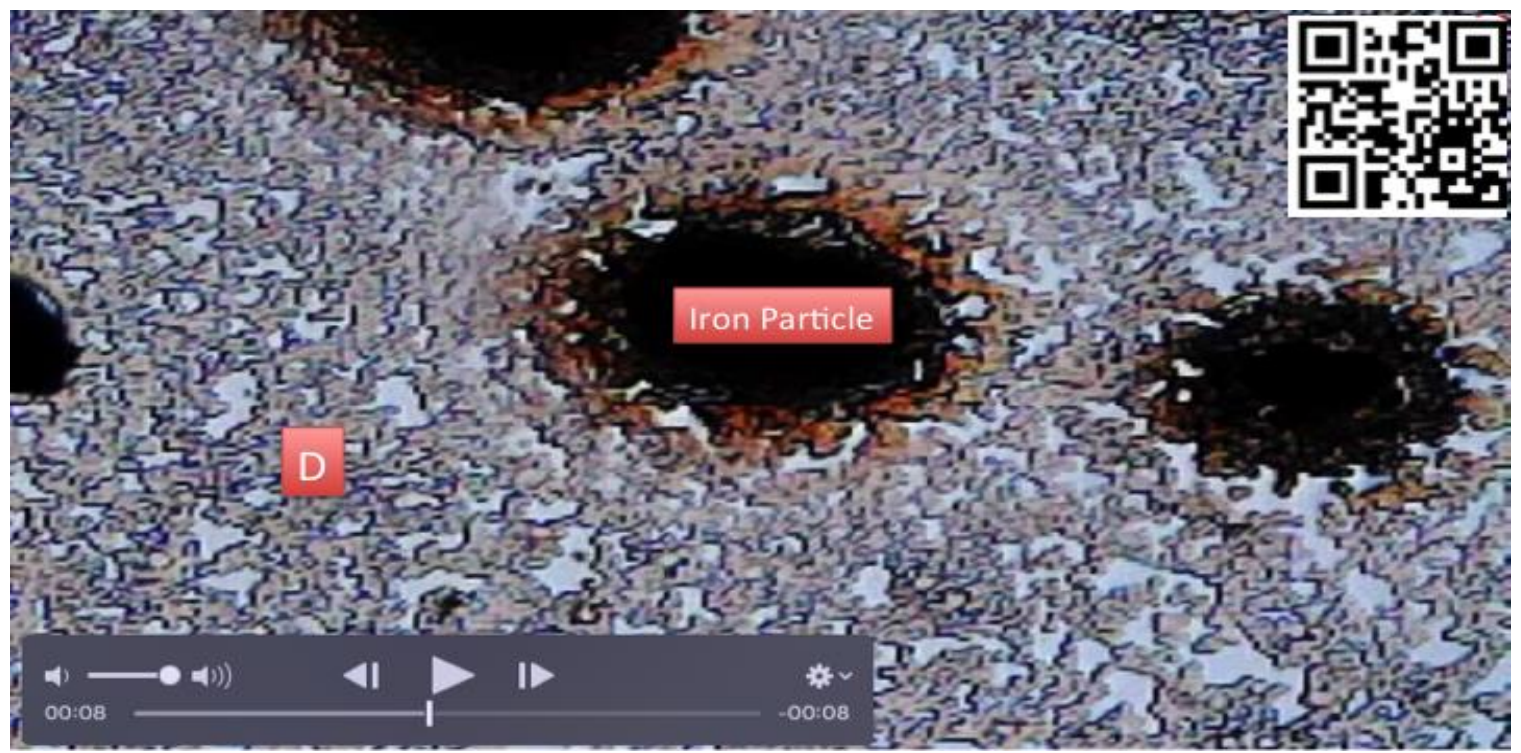

Figure 5: Video frame at 00:08”. Iron Metal Filings in TIBS. Demonstration of darker background (D) just prior to end of coagulation cascade. For additional details of the light radiation emission (at 00.09") post coagulation please link to: https://youtu.be/xaDkVKEGowQ or Scan QR Code in top right corner of image.

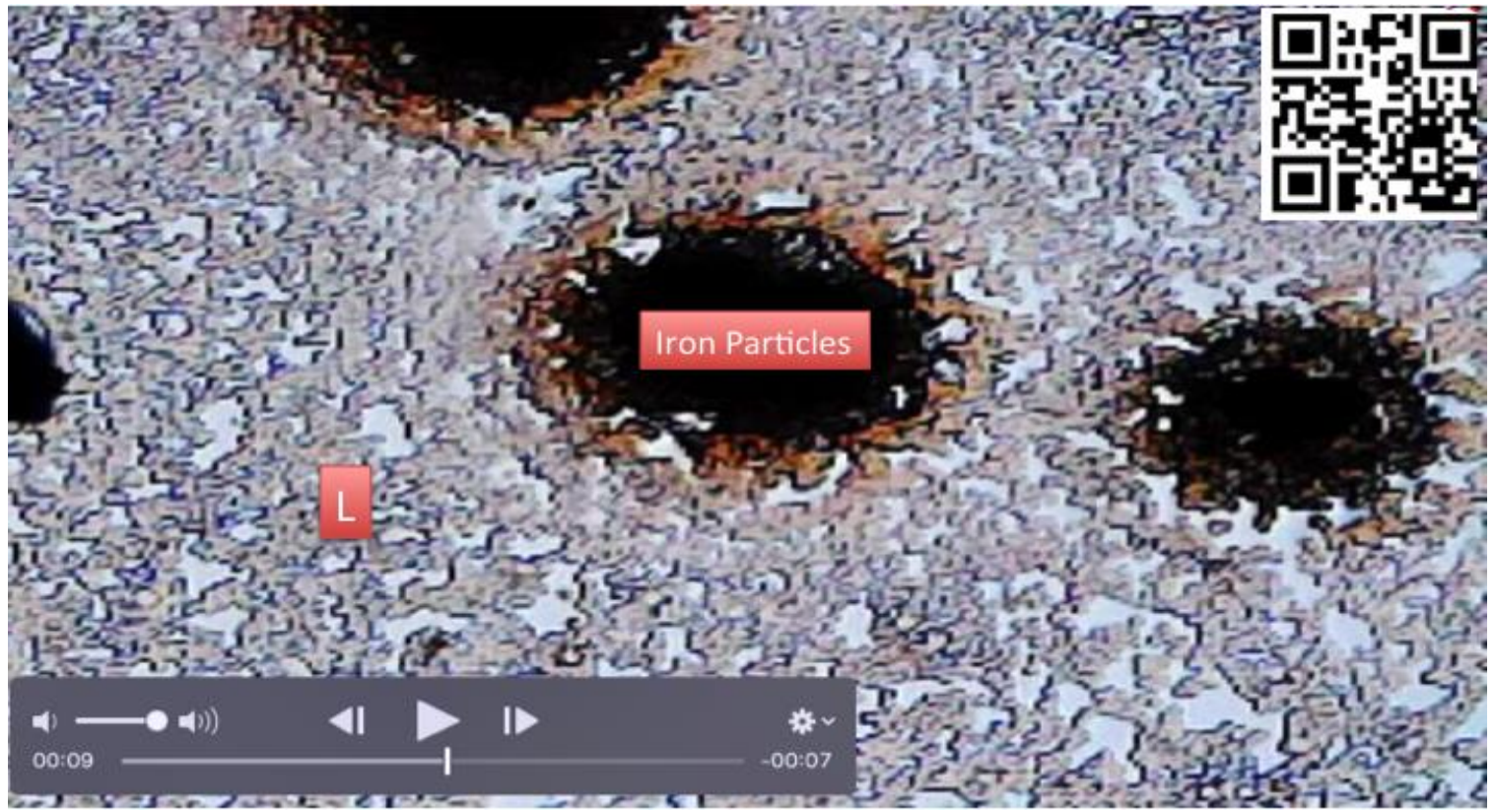

Figure 6: Video frame at 00:09”. Iron Metal Filings in TIBS. Demonstration of light radiation emission seen post end of coagulation. For additional details of the light radiation emission post coagulation please link to: https://youtu.be/xaDkVKEGowQ or Scan QR Code in top right corner of image. 
Example of iron particles in TIBS attracting RBCs

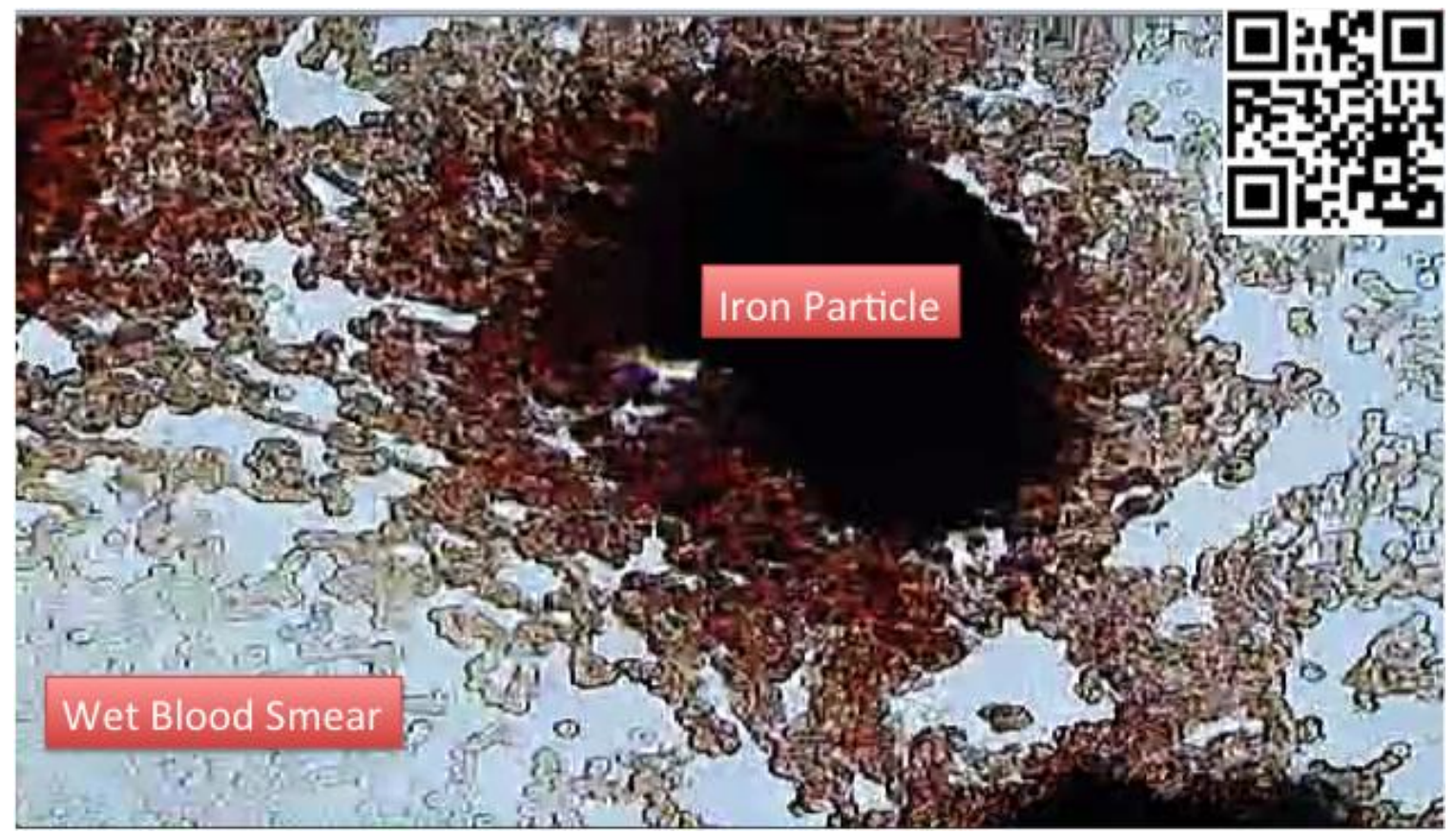

Figure 7: Showing several iron particles on a TIBS attracting and competing for RBCs. Please link to: https://youtu.be/M8dKiT90Bx4

Or Scan QR Code on right top of image.

\section{Supplemental experiment confirming Iron Filing Effect on TIBS}

To appreciate light phenomenon at end of coagulation cascade, please move cursor back and forth at a faster speed.

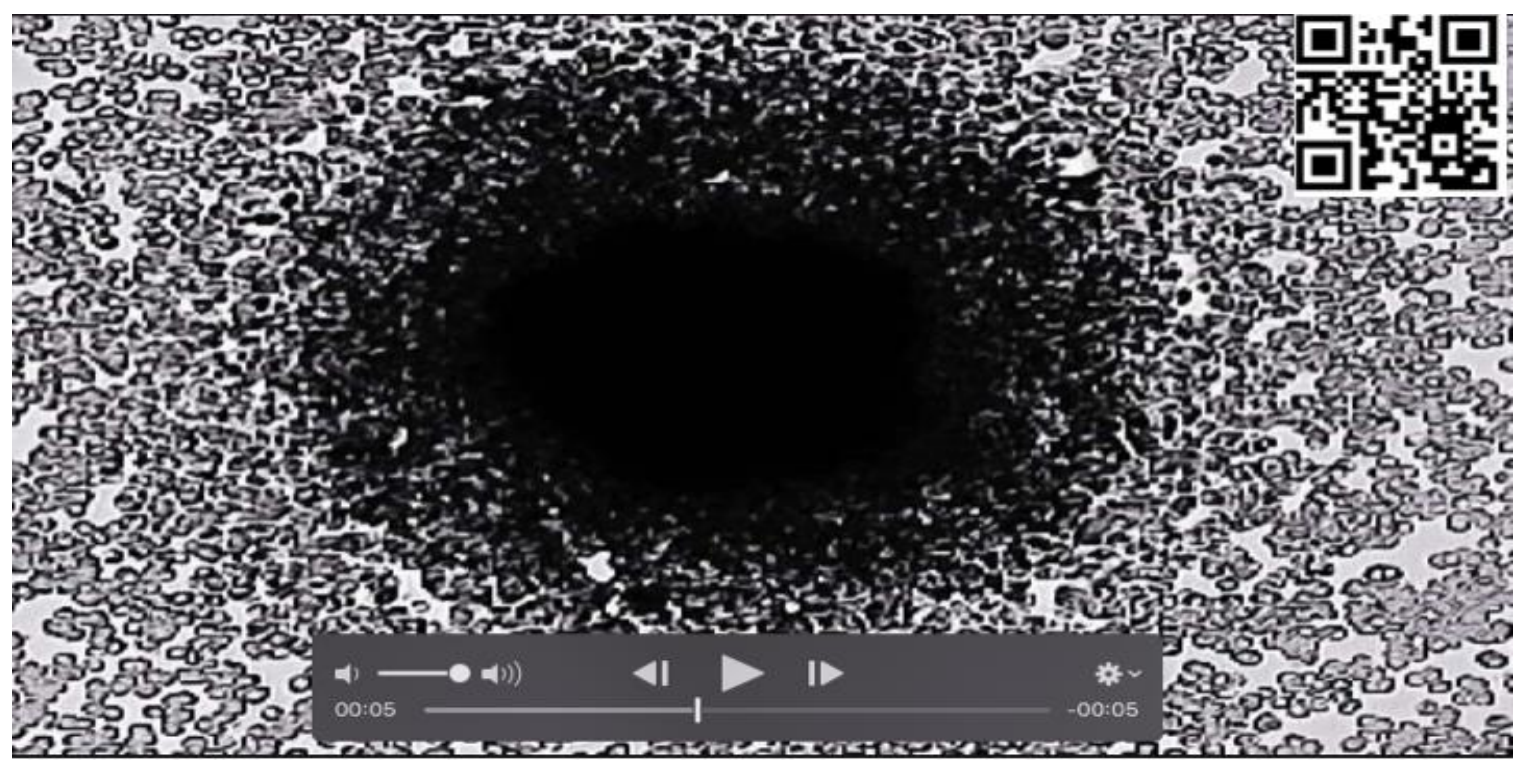

S-1: Unpublished. Video-frame at 00:05" of recording -Prior to end of contraction. (coagulation) For details link to: https://youtu.be/hy1Of-wAcS4 Or scan QR Code on top right corner of image. 


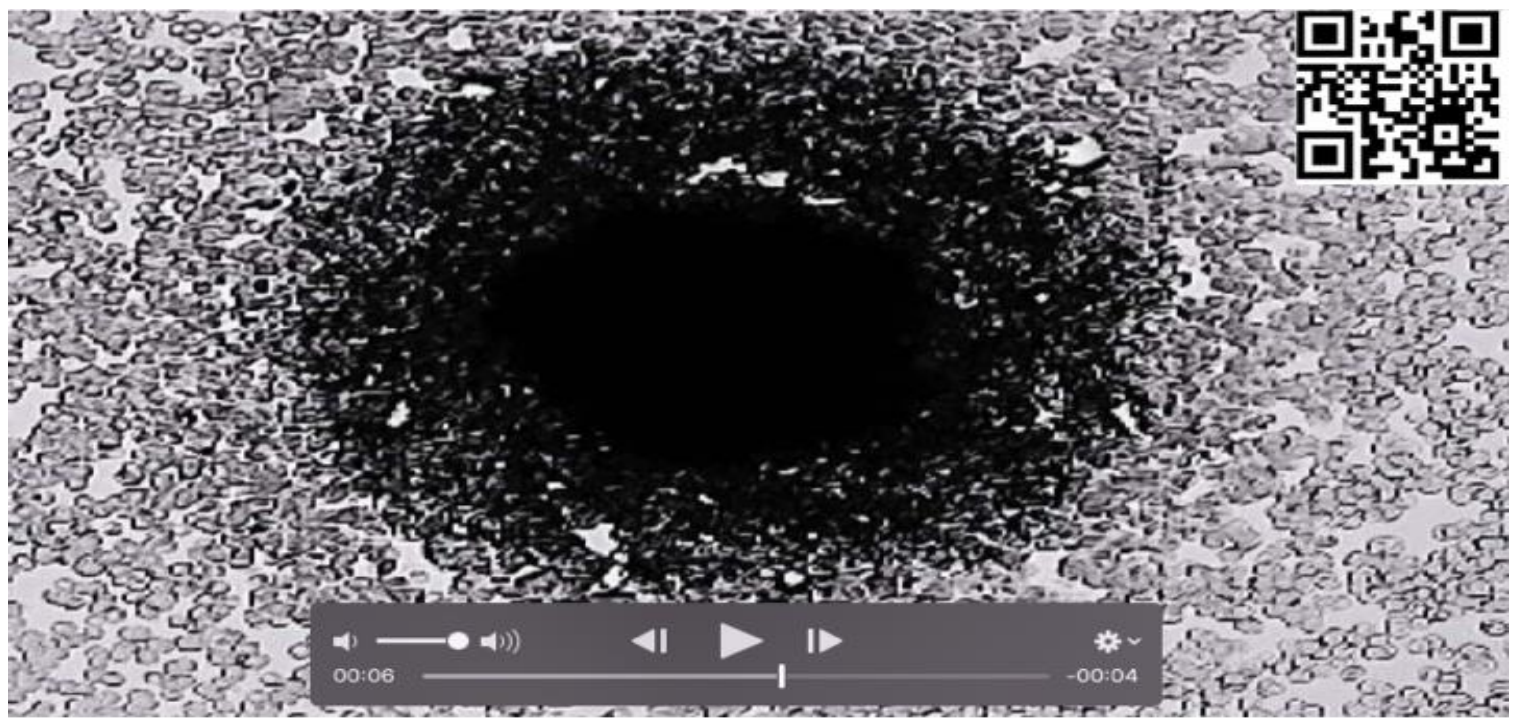

S-2: Videoframe at 00:06" of recording- (Mid-way into contraction. (coagulation) Temporary out of focus (vertical movement). Link to:

https://youtu.be/hy1Of-wAcS4 or Scan QR Code in upper right corner.

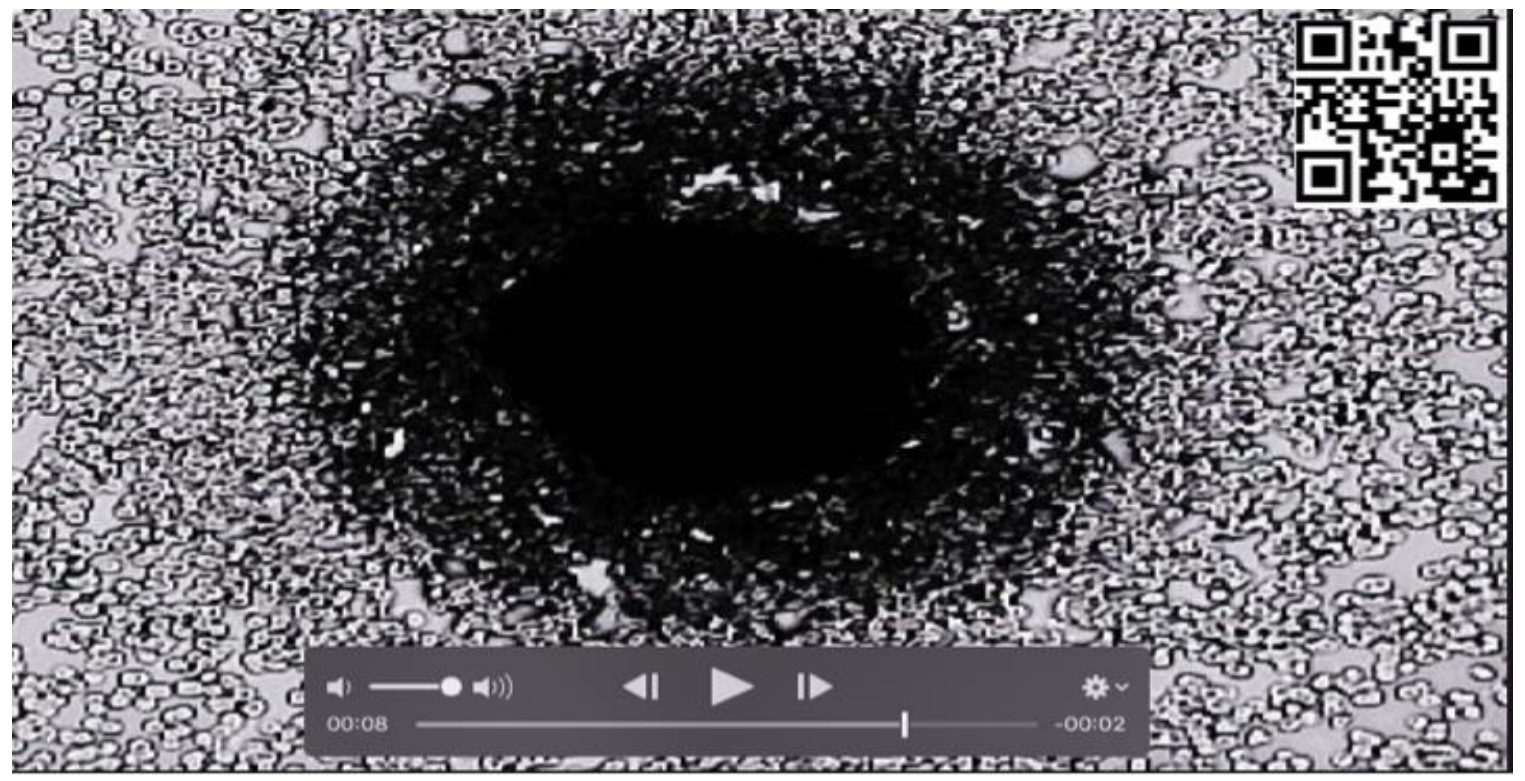

S-3: Videoframe at 00:08" of recording. End of contraction- Light rays after full contraction. (End of coagulation). Link to:

https://youtu.be/hy1Of-wAcS4. Or Scan QR Code in upper right corner.

\section{Supplemental Image from Previous Experiments Biomagnetism Triggers Blood Coagulation}

Images of a biological material (human hair shafts) unilateral biomagnetism had been documented in triggering fibrin (clot) formation. In the same image the contralateral side void of biomagnetism did not trigger clot formation, please see reproduced image from previous research below. 
Demonstration of hair shaft unilateral bio-magnetism attracting and deforming RBCs

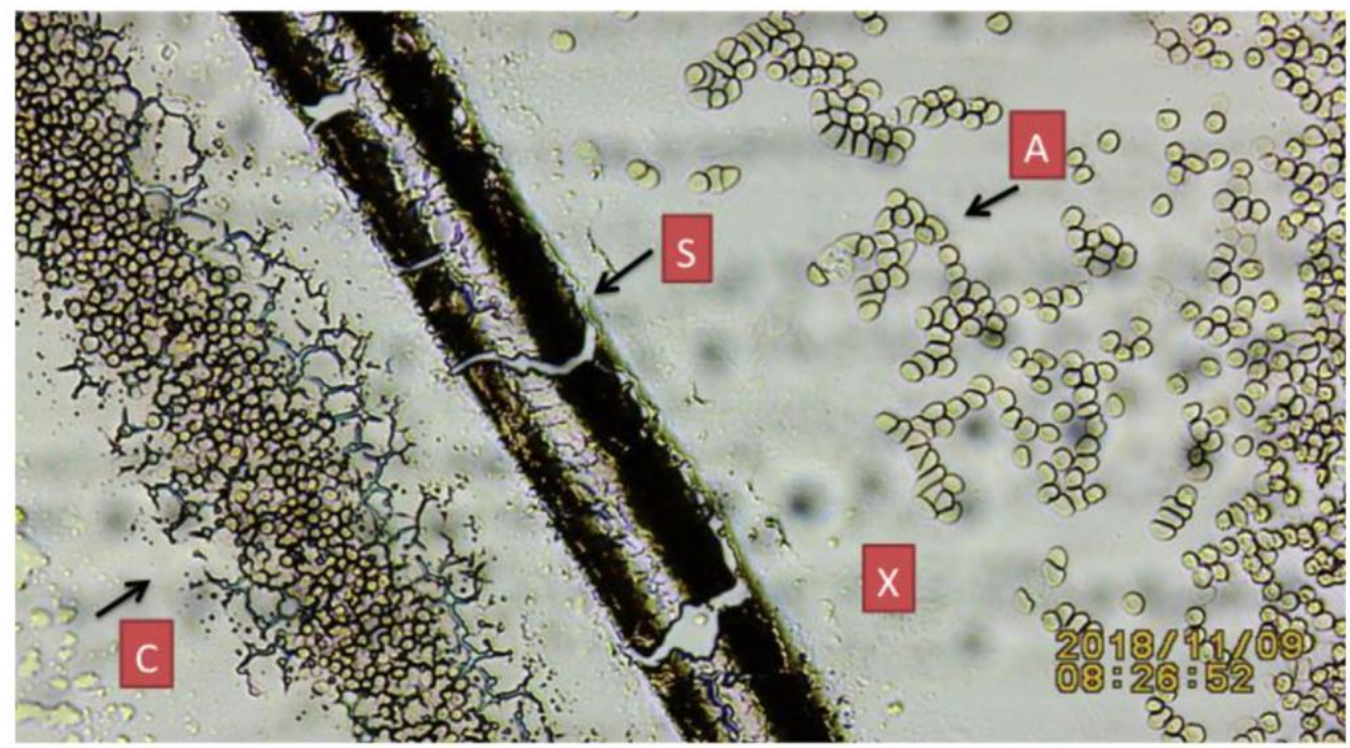

Microphotograph depicts hair shaft outline after hair proper removed from TIBS. A= RBCs, Agglutinated and in Rouleau Formation. $\mathrm{S}=$ Hair shaft. $\mathrm{C}=$ Coagulated blood, denoting fibrin formation in side void of biomagnetic fields. $\mathrm{X}=$ Diamagnetic zone.

Image reproduced from: Embi AA. (2018) Hair and blood endogenous low level biomagnetic fields crosstalk effects on fibrin inhibition and rouleau formation. IJGR, 6(11), 200-208. https://doi.org/10.5281/zenodo. 1845985.

\section{Additional Figures}

Demonstration of RBCs accumulation underneath a paramagnetic iron particle after removal of Iron metal filing in TIBS: Showing post coagulation clumping of RBCs

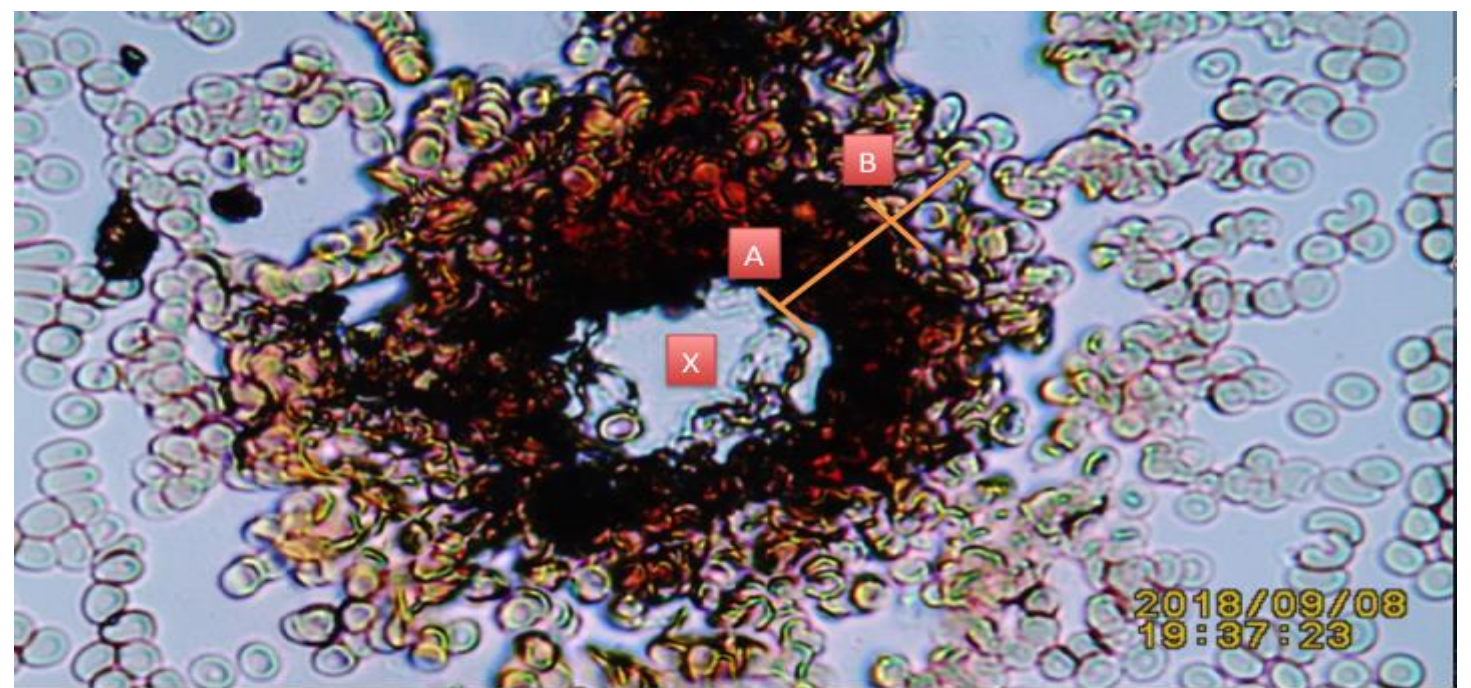

Figure 8: Iron particle removed via wooden toothpick, showing "crater" at center of image. The void observed is hypothesized as resulting from energy released at end of the coagulation process. $\mathrm{X}=$ Theorized center of released energy. A B Line $=$ Greater circumferential accumulation of 
compressed RBCs. Demonstrating the after effects of the end of coagulation cycle, where blood tissue was compressed due to the paramagnetic iron metal particles.

Similar example of RBC clumping or accumulation due to paramagnetic attraction. Image denotes post iron particle removal.

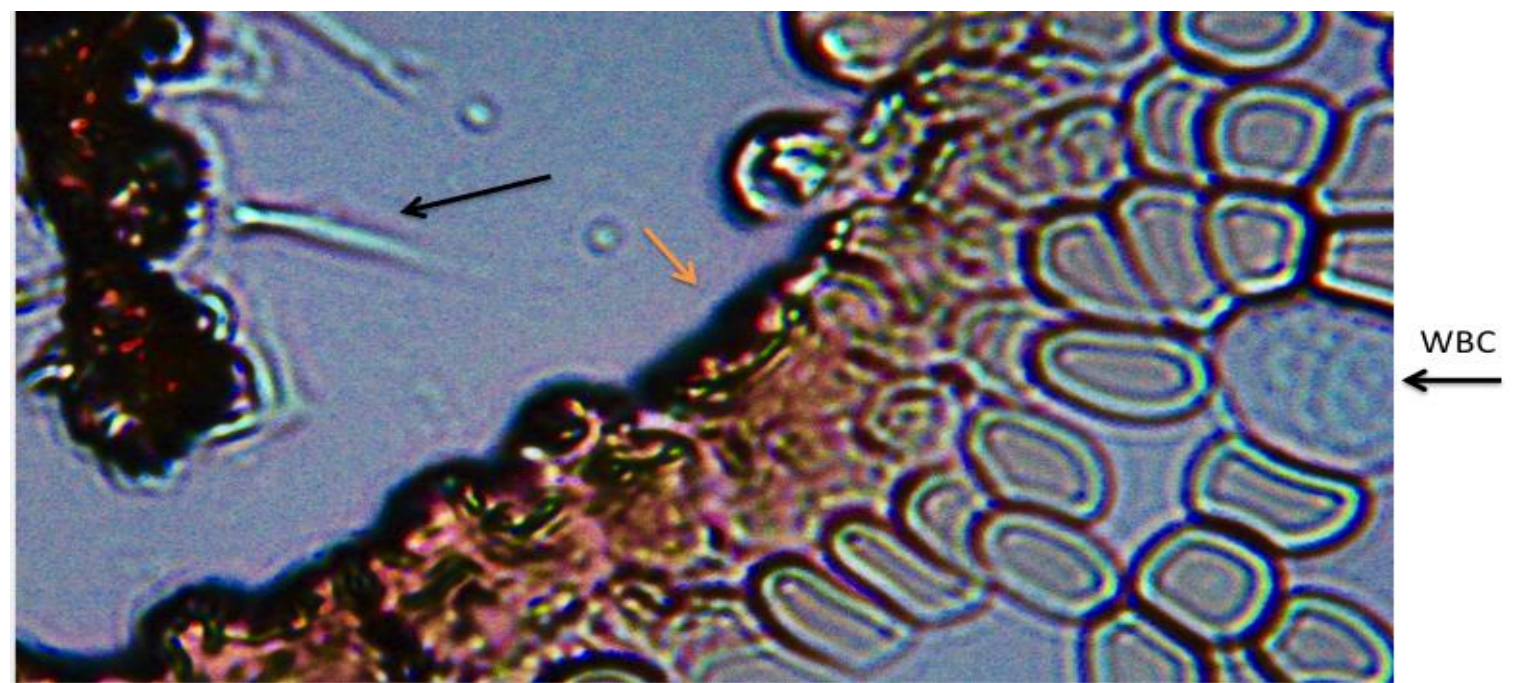

Figure 9: Compressed RBCs. Amplified inner core after removing iron particle- Black Arrow= Teardrop deformed RBC. Orange Arrow: Compressed RBCs. -

Similar to the iron particles examples, graphite is known to behave as a metal. Below is also documentation of light radiation emitted post evaporation.

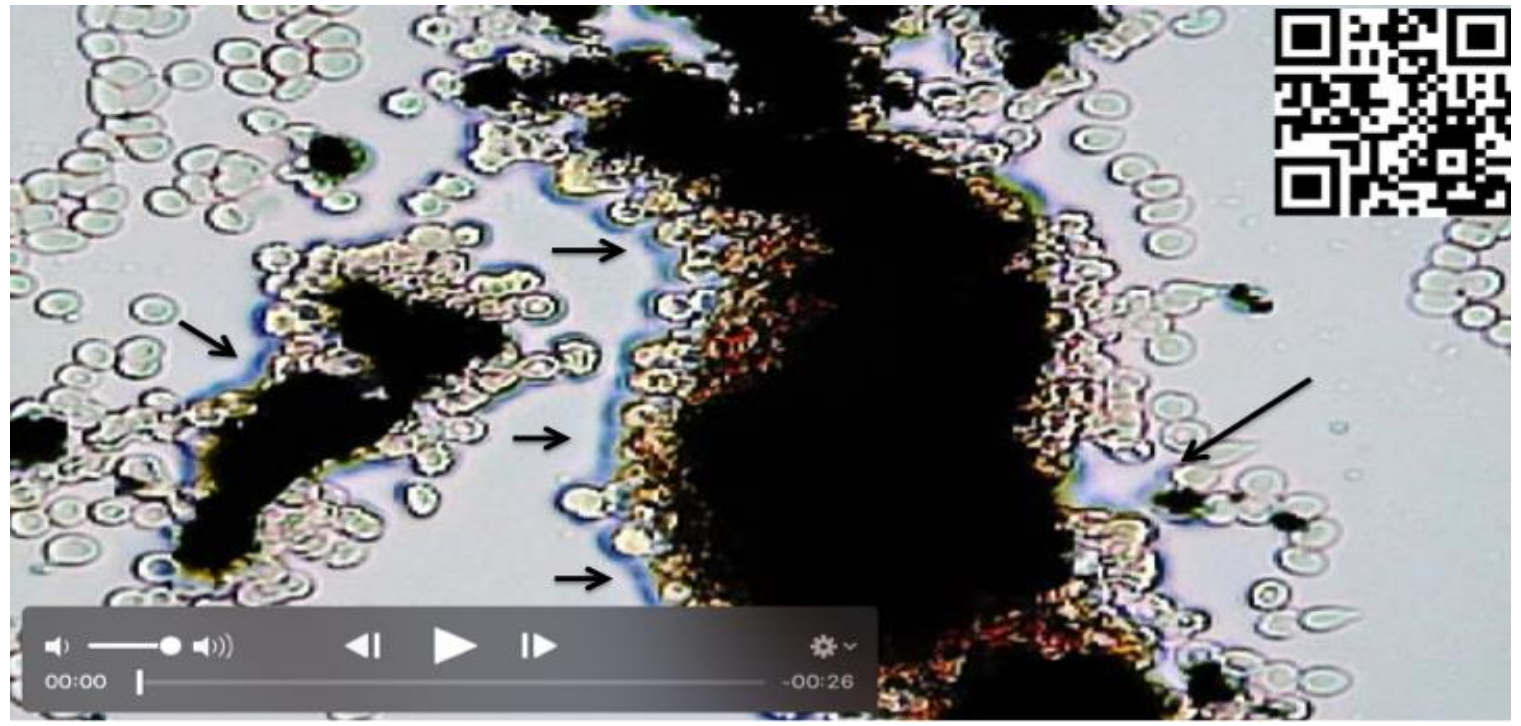

Figure 10: Frame 00:35". Fresh RBC smear on slide (TIBS) showing graphite particles attracted and also repulsed by the graphite. Notice sudden image change in Fig 8 denoting the end of blood coagulation. Black arrow= Blue shadow theorized to be from water still present in TIBS.

Orange arrow $=\mathrm{RBC}$. Please link to:

https://youtu.be/Sfu2MfO9RWQ or Scan QR Code on top right of Fig 7 image. 


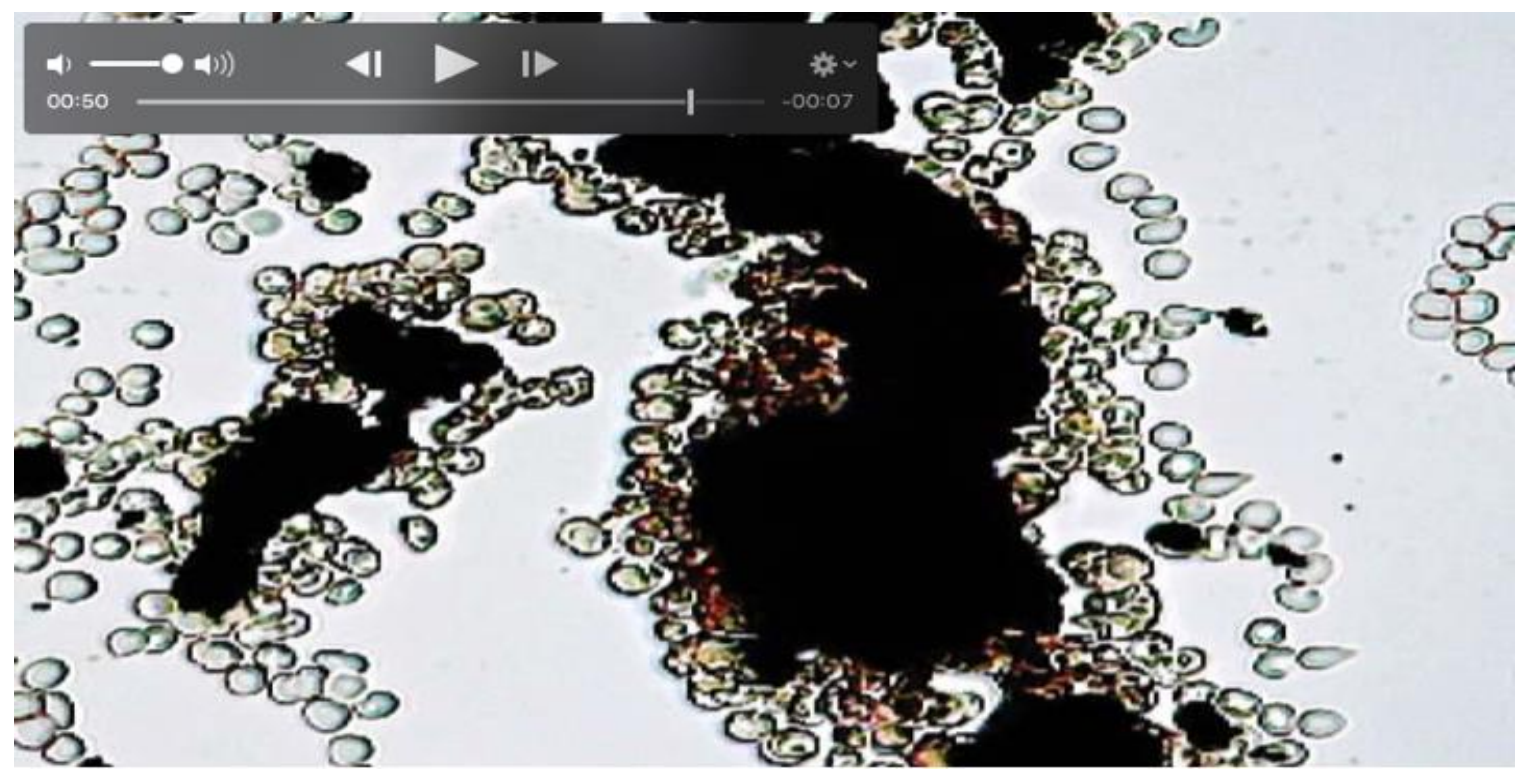

Figure 11: Frame 00:50”. Blood coagulation has ended. The graphite particles appear to shrink; theorized is that the coagulated blood under the graphite particles has now shrunk (end of coagulation) thus shifting the microscope depth of field; and lighter (light radiation increase)

Please link to: image.

https://youtu.be/Sfu2MfO9RWQ or Scan QR Code on top right of Fig 7 image.

\section{Definition of Terms}

To aid the reader, the following definitions are included:

Biological Material: Are present inside living organisms. They do not contain any metal, ceramic, or synthetic polymer material and have the ability to repair themselves,

Biomaterial: Is any combination of natural and synthetic substances (other than drugs) that can be used in the treatment of functions of the body.

TIBS: Acronym for "Temporary In Vivo Blood Smear"

Limitations: It could be argued that only qualitative images are presented. The measuring of electrical discharges are suggested in future experiments.

\section{Discussion}

Due to its medical implications, the human blood coagulation cascade has been extensively researched from a Biochemistry and Molecular Perspective. The temporary preservation of in vivo physical properties of a biological material, such as whole human blood tissue smeared on a glass slide (TIBS) allowed for a demonstration of biological Material cross-talk between endogenous or exogenous magnetically active materials. Demonstrated was RBC deformations and a triggering effect in initiating the blood coagulation cascade. Results from that research were inferred by static images denoting physical changes. In this manuscript, the above findings are now expanded to include video-recordings of in vivo TIBS preparations where whole blood was in direct contact with highly electrically conductive materials such as metal iron filings and graphite particles $(10,11)$. Blood tissue was documented being attracted by bio-magnetic active exogenous materials. The qualitative findings show for the first time light radiation emitted at the end of the coagulation 
cascade; which happens to coincide with fibrinogen induced fibrin burst. It could be hypothesized that the light emissions could be attributed to a piezoelectric effect from cells clumping due to the magnetic attraction of iron or graphite particles (Figs. 8,9,10,11). This is supported by a previously published experiment demonstration of a piezoelectric effect; where light emission was attributed to the sudden compression of biological material (eukaryotic cells) shifting in laminar flows (12). Also hypothesized is the optical absorption by water phenomenon (13) on the TIBS slide. The methodology herein presented could facilitate and expand, albeit with the proper instrumentation for the evaluation of blood clotting disorders.

\section{Ethics Statement}

In compliance. The blood drops tested were from main author.

\section{Conflict of Interests}

None to declare.

\section{Funding Sources}

None, Research, Ideas, drafting, writing and self-funded by main author.

\section{References}

[1] Embi AA. "BIOMAGNETISM AS FACTOR IN RED BLOOD CELLS DEFORMATION." International Journal of Research - Granthaalayah, 20186 (12), 46-57. https://doi.org/10.5281/zenodo.2528404.

[2] Embi AA. Hair and blood endogenous low level biomagnetic fields cross-talk effects on fibrin inhibition and rouleau formation. IJGR, 2018 6(11), 200-208. https://doi.org/10.5281/zenodo. 1845985.

[3] Embi AA. Expanding the role of magnetic fields in red blood cells deformations: Demonstration of paramagnetic and diamagnetic fields. IJRG, 2019 7(2), 214-220. https://doi.org/10.5281/zenodo.2587463

[4] Wolberg, A. S., \& Campbell, R. A. Thrombin generation, fibrin clot formation and hemostasis. Transfusion and apheresis science: official journal of the World Apheresis Association: official journal of the European Society for Haemapheresis, 2008 38(1), 15-23. doi:10.1016/j.transci.2007.12.005

[5] Smith, S. A., Travers, R. J., \& Morrissey, J. H. How it all starts: Initiation of the clotting cascade. Critical reviews in biochemistry and molecular biology, 2015 50(4), 326-336. doi:10.3109/10409238.2015.1050550

[6] Bates S.M. Coagulation Assays. Circulation; 112:e53-e60. doi: 10.1161/CIRCULATION AHA. 2005104.478222

[7] Sperling C., Fischer M., Maitz M.F., Werner C. Blood coagulation on biomaterials requires the combination of distinct activation processes. Biomaterials. 2009; 30: 4447-4456. doi: 10.1016/j.biomaterials.2009.05.044

[8] Weisel, J.W., \& Litvinov, R.I. Mechanisms of fibrin polymerization and clinical implications. Blood, 2013121 10, 1712-9

[9] Center for Disease Control. USA. Suggested technique for BloodSmear. https://www.cdc.gov/dpdx/diagnosticprocedures/blood/specimenproc.html 
[10] Ragheb, T., \& Geddes, L.A. Electrical properties of metallic electrodes. Medical and Biological Engineering and Computing, 1990 28, 182-186.

[11] Liu, N., Chortos, A., Lei, T., Jin, L., Kim, T.R., Bae, W., Zhu, C., Wang, S., Pfattner, R., Chen, X., Sinclair, R., \& Bao, Z. Ultratransparent and stretchable graphene electrodes. Sci Adv. 2017 Sep 8;3 (9):e1700159. doi: 10.1126/sciadv.1700159.

[12] Embi AA. The Dual Effect on Eukaryotic Cells of Bursting Air Bubbles Shockwaves Induced Piezoelectric Effect as Additional Factor in Extracorporeal Focused Very Intense Pressure Pulse Cancer Cells Treatments. Acta Scientific Cancer Biology 2019 3. 10 : 24-28.

[13] C. K. N. Patel and A. C. Tam, "Optical absorption coefficients of water," 1979 Nature London 280, 302-304.

*Corresponding author.

E-mail address: embi21@ att.net 